\title{
Hepatoma-Derived Growth Factor Is Expressed after Vascular Injury in the Rat and Stimulates Smooth Muscle Cell Migration
}

\author{
JILL V. NARRON, TAMARA D. STOOPS, KURT BARRINGHAUS, MARTIN MATSUMURA, AND ALLEN D. EVERETT \\ Department of Pediatrics [J.V.N, T.D.S., A.D.E.], Department of Internal Medicine [K.B., M.M.], Cardiovascular Research Center
[M.M., A.D.E.], University of Virginia Health System, Charlottesville, Virginia, 22908; Department of Pediatrics [A.D.E.],
} Johns Hopkins Hospital, Baltimore, Maryland, 21287

\begin{abstract}
The role of hepatoma-derived growth factor (HDGF), a novel nuclear-targeted vascular smooth muscle cell (SMC) mitogen in vascular injury is unknown. We hypothesized that HDGF plays a role in SMC proliferation and migration in formation of the neointima after balloon injury of the rat carotid. Using co-immunohistochemical staining, HDGF and proliferating cell nuclear antigen (PCNA) were co-expressed in $80 \%$ of nuclei of neointimal cells $7 \mathrm{~d}$ post carotid balloon injury with HDGF. The HDGF-positive medial and neointimal cells were smooth muscle actin negative and therefore likely represented a subgroup of SMC that have undergone phenotypic switching. Utilizing modified Boyden chamber migration assays, adenoviral-expressed HDGF in mouse SMC increased migration 10 -fold (20 versus 2 ). HDGF gene silencing reduced both SMC proliferation and migration. In conclusion, HDGF is highly expressed in the media and neointima post balloon injury, a SMC mitogen and positive regulator of cell migration. We speculate that HDGF is involved in the SMC proliferative and migratory response to injury resulting in neointimal formation. (Pediatr Res 59: 778-783, 2006)
\end{abstract}

$\mathrm{N}^{\mathrm{e}}$ eointimal hyperplasia and resultant restenosis are welldescribed entities following vascular injury and represent a significant source of morbidity in patients undergoing balloon angioplasty for occlusive vascular disease (1), but the underlying mechanisms are not completely understood (2). One model of the response to injury suggests that denudation of endothelial cells after balloon angioplasty triggers the release of endogenous factors leading to SMC proliferation and migration, a subsequent change in the composition of the extracellular matrix, and formation of the neointima (2). Agents implicated in this process include proto-oncogenes (c-fos, c-jun, and c-myc), mitogens such as b-FGF, cytokines such as interleukins and tumor necrosis factor (TNF), and growth factors such as platelet-derived growth factor (PDGF) (3). PDGF, b-FGF, and other growth factors are known to stimulate SMC proliferation and migration with resultant neointimal hyperplasia $(3,4)$.

HDGF represents the first member identified of a new family of nuclear-targeted growth factors without homology to any other group (5). It was initially isolated as a secreted

Received December 13, 2005; accepted February 1, 2006

Correspondence: Allen D. Everett, M.D., Johns Hopkins Hospital, 600 N. Wolfe St., Baltimore, MD 21287; e-mail: aeveret3@jhmi.edu

Supported by the National Heart Lung and Blood Institute, National Institutes of Health (grant R01-HL069938 to ADE).

DOI: 10.1203/01.pdr.0000219299.24435.4f heparin binding growth factor from the conditioned media of $\mathrm{HuH}-7$, a human hepatoma cell line in culture, and its role in cardiovascular growth is unknown (5). Prior studies have shown that HDGF is a potent SMC mitogen in culture (6) and HDGF is expressed in nuclei of neointimal cells at $14 \mathrm{~d}$ in response to vascular injury (6). However, HDGF's onset of expression after injury and its relationship to SMC proliferation are unknown. In the present study, we determined that HDGF is expressed in the media within $3 \mathrm{~d}$ of vascular injury and plays a role both in SMC migration and proliferation, important pathways in neointima formation.

\section{MATERIALS AND METHODS}

Rat carotid injury model. Male 300-g Sprague-Dawley rats (Harlan Bioproducts for Science Inc., Indianapolis, IN) underwent carotid artery balloon injury as described previously (6). All protocols conformed to institutional guidelines. After pressure perfusion with $4 \%$ paraformaldehyde in cold PBS, the injured left and control right carotids were removed and postfixed in $4 \%$ paraformaldehyde for $2 \mathrm{~h}$, dehydrated in a graded alcoholxylene series, and paraffin embedded for thin sectioning. Arteries were harvested at 3 and $7 \mathrm{~d}$ post-balloon injury.

Cell culture. Primary cultured mouse vascular smooth muscle cells were grown in Dulbecco's modified Eagle medium/F-12 (Invitrogen, Carlsbad, CA) supplemented with $1 \times$ Na pyruvate and $10 \%$ FBS (fetal bovine serum) in a $5 \% \mathrm{CO}_{2}$ incubator at $37^{\circ} \mathrm{C}(6)$.

HDGF immunohistochemistry. Rat carotid injury slides were blocked with normal goat serum (Vector Laboratories, Burlingame, CA) for $20 \mathrm{~min}$ at room temperature, washed, and incubated with HDGF affinity-purified polyclonal antibody at a dilution of 1:1000 (6,7). After washing with PBS/0.1\% Tween-20, HDGF immunodetection was performed using the ABC-AP method (Vector Laboratories) with Vector Red as a chromogenic substrate. After HDGF staining, slides were immersed in $10 \mathrm{mM}$ citrate buffer, $\mathrm{pH} \mathrm{6}$, at $95^{\circ} \mathrm{C}$ for $20 \mathrm{~min}$. After cooling, slides were blocked with normal horse serum (Vector Laboratories) for $20 \mathrm{~min}$ at room temperature, washed, and then incubated with a monoclonal anti-PCNA antibody (1:200; DAKO, Carpinteria, CA). PCNA immunodetection was performed using an Oregon Green conjugated anti-mouse antibody (Molecular Probes, Eugene, OR) diluted $1: 1000$.

HDGF and $\alpha$ SMA co-immunostaining was performed as above without the citrate antigen retrieval. After immunodetection of HDGF with Vector Red, sections were briefly washed with PBS/Tween and incubated with a mononclonal $\alpha$ SMA antibody (1:1000; Sigma Chemical Co., St. Louis, MO) for $1 \mathrm{~h}$ followed by incubation with the Oregon Green secondary antibody as described above. Slides were mounted (Vectashield; Vector Laboratories), visualized, and photographed with an Olympus BX5OWI Fluoview confocal

Abbreviations: $\boldsymbol{\alpha S M A}$, alpha smooth muscle actin; b-FGF, basic fibroblastderived growth factor; BrdU, bromodeoxyuridine; GFP, green fluorescent protein; HDGF, hepatoma-derived growth factor; MOI, multiplicity of infection; PCNA, proliferating cell nuclear antigen; shRNA, short hairpin interfering RNA; SMC, smooth muscle cell 
microscope or a Nikon Eclipse E400 microscope (Nikon, Tokyo, CA) coupled to a Q-Imaging Micropublisher (Q-Imaging, Burnaby, Canada) digital camera.

HDGF-expressing adenovirus. A replication-deficient type-5 adenovirus was prepared as a GFP-HDGF fusion protein as previously described (7) using the methods of Hardy et al. (8). The appropriate viral dose for mouse SMC was determined by infecting cells in complete growth media for $4 \mathrm{~h}$ with increasing MOI and then detecting HDGF expression by immunoblotting for GFP a total of $24 \mathrm{~h}$ after infection.

BrdU ELISA. BrdU ELISA as a measure of cell proliferation was performed as described previously (7). Briefly, SMC cell proliferation using the Colorimetric Cell Proliferation ELISA (Roche Molecular Biochemicals, Indianapolis, IN) was used. In each well of a 96-well, clear, tissue culturetreated flat-bottom culture plate (Corning Inc. Corning, NY), 3200 mouse SMC in complete growth media were plated, allowed to attach, and infected with Ad5-GFP-HDGF or Ad5-GFP at a MOI of 25 for $4 \mathrm{~h}$. The virus was removed and the cells incubated in complete growth media for $14-16 \mathrm{~h}$ to recover. Stabilized cells were then transferred to serum-free media for $8-12 \mathrm{~h}$ and then incubated overnight with $10 \mu \mathrm{M}$ BrdU (Roche Molecular Biochemicals). Each condition was performed in pentuplicate per assay. BrdU uptake was determined using a $\mu$ Quant plate reader (Bio-Tek Instruments, Highland Park, VT). Blanks include culture media + BrdU (Roche Molecular Biochemicals) and anti-BrdU-POD (peroxidase, Roche Molecular Biochemicals). Controls include noninfected cells + culture media and anti-BrdU-POD. Results for each condition were averaged and compared using one-way ANOVA and $t$ test between individual groups.

Modified Boyden chamber migration assay. Mouse SMC infected (MOI 25 ) with adenovirus to express GFP or GFP-HDGF were added to 24-well, $8 \mu \mathrm{m}$ pore size BioCoat cell culture inserts (Becton Dickinson, Bedford, MA) at 50,000 cells/insert in complete growth media and allowed to attach for $6 \mathrm{~h}$. The media from the inserts and wells was replaced with serum-free media and incubated for $12 \mathrm{~h}$ to allow migration into serum containing media. After allowing migration, the cells were washed with PBS and fixed with $2 \%$ paraformaldehyde in PBS for $20 \mathrm{~min}$ at room temperature. Cells adherent to the upper side of the insert membrane (nonmigrating) were removed by gentle scraping with a cotton swab. The membrane was removed from the insert and placed on a microscope slide bottom side up with mounting medium containing DAPI (4'-6-diamidino-2-phenylindole, Vectashield; Vector Laboratories). Control conditions included noninfected SMC with serum-free media or $10 \%$ FBS in the wells and SMC infected with GFP control adenovirus at a MOI of 25. The assay was performed in triplicate for each condition and then repeated. Five random high-power fields per membrane were photographed using a Nikon Microphot-SA equipped with a Hamamatsu C4742 chargecoupled device camera driven by OpenLab 2.0.6 (Improvision, Lexington, MA). The number of GFP-positive migrating cells per field was counted, averaged, and compared by $t$ test.

HDGF shRNA lentivirus. Lentiviral mouse- and human-specific HDGF shRNA using the system described by Rubinson et al. (9) were produced. Briefly, the mouse (1443-AGGCTACCATCCATCCATAAA) and human (2107-GTGTCATTTCTCATCCACATA) specific HDGF sequences were ligated into a lentiviral shuttle vector and packaged in the human 293-T packaging cell line. The resultant lentivirus expresses the shRNA under control of the U6 promoter and GFP downstream of the shRNA under control of an IRES (internal ribosomal entry sequence) sequence. In all studies, lentiviral infection resulted in $100 \%$ of the cells expressing GFP as a marker of infection efficiency. Mouse SMC were incubated with media containing lentivirus with mshRNA or hshRNA for $6 \mathrm{~h}$ with rotation followed by removal of the virus containing media and returned to standard culture conditions. SMC were harvested $72 \mathrm{~h}$ after infection for Western blotting (HDGF) or replated for cell proliferation (BrdU incorporation) or migration (modified Boyden chamber assays).

\section{RESULTS}

Mapping HDGF expression with PCNA after vascular injury. SMC proliferation is an integral step in the expected cellular response to vascular injury. To determine whether HDGF expression is linked to neointimal formation and SMC proliferation, paraffin-embedded sections of injured left and control right rat carotid arteries harvested at 3 and $7 \mathrm{~d}$ postballoon injury were double-immunostained for HDGF and PCNA, a marker of DNA synthesis. As shown in Figure 1, without injury, there is no expression of HDGF or PCNA in the carotid artery wall, illustrating the low turnover rate of SMC in the arterial wall. Only background, nonspecific autofluorescence of the arterial wall is seen. At $3 \mathrm{~d}$ after injury, no neointima had developed, but HDGF was highly expressed in medial cells as a nuclear protein. Compared with HDGF, PCNA expression was low, with co-expression in only one medial cell (Fig. 1, arrow). By 7 d, a highly proliferative neointima had formed with several populations of cells observed. Numerous cells in the neointima expressed PCNA, which appeared to be limited exclusively to this area of the vessel wall. In contrast, HDGF continued to be expressed in a modest number of medial cells and, similar to PCNA, was also largely present in cells of the proliferating neointima. Many neointimal cells co-expressed PCNA and HDGF, but there were clearly cells that stained for either HDGF or PCNA and did not overlap. When PCNA and HDGF cells were counted, $80 \%$ of PCNA-positive cells were also HDGF-positive. Although PCNA-positive cells were not observed in the medial layer, many HDGF-positive cells were present within the media. Although HDGF has been described as a secreted protein in vitro (5), at any of the time points examined after injury, HDGF was only observed in the nucleus. Expression of HDGF was exclusive to medial and neointimal cells with no
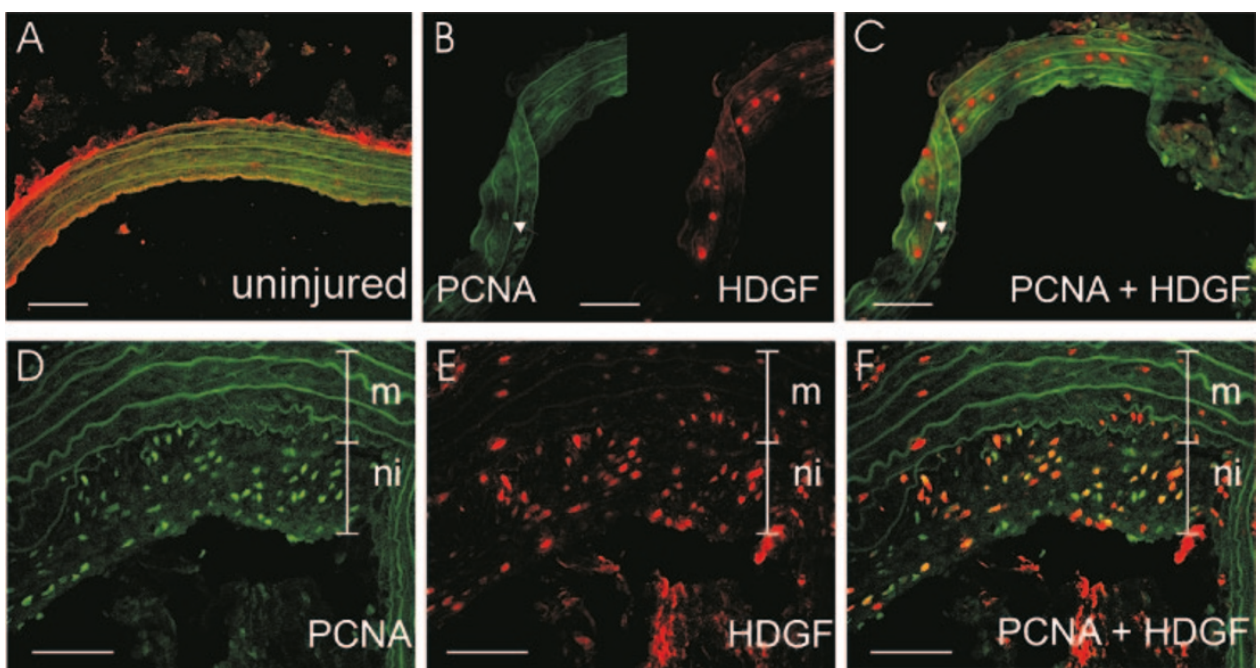

Figure 1. HDGF is expressed before neointimal formation and co-expressed with PCNA in the neointima after rat carotid balloon injury. Representative sections of rat carotid artery co-immunostained for HDGF (red) and PCNA (green) in a sham-injured carotid $(A)$ and carotids $3 \mathrm{~d}(B$ and $C)$ and $7 \mathrm{~d}$ $(D-F)$ after balloon injury. $M$, media; $n i$, neointima. Arrows indicate PCNA and HDGF co-localization at $3 \mathrm{~d}$. Magnification bars $=50 \mu \mathrm{m}$. 
expression observed at any time point in the adventitia. We did not observe cytoplasmic or basement membrane localization of HDGF in the carotid artery after injury indicative of HDGF secretion.

HDGF expression represents a subpopulation of SMC. We observed (Fig. 1) that HDGF expression in the carotid artery after injury was limited to expression in the medial and neointimal layers. To examine whether HDGF expression identifies a subpopulation of SMC that have undergone phenotypic switching in response to injury, we co-immunostained carotid artery sections $7 \mathrm{~d}$ after injury for HDGF and $\alpha$ SMA as a marker for SMC expressing a normal contractile differentiated phenotype. As shown in Figure 2, after 7 d of injury, HDGF-expressing cells are abundant in the large neointima $(N, r e d)$. Occasional medial cells were seen to express HDGF, but none were observed in the adventitia. When the total number of medial nuclei were counted (DAPI stained) and compared with the number of HDGF positive nuclei, $97 \%$ of neointimal nuclei were positive for HDGF. Co-immunostaining for HDGF and $\alpha$ SMA (green) in the same section allowed us to examine whether HDGF-positive cells express a marker of differentiated SMC. We observed $\alpha$ SMA expression as dense cytoplasmic bundles predominately in cells of the medial layer consistent with fully differentiated SMC. Neointimal cells expressed very little $\alpha$ SMA, with most of the $\alpha$ SMA expression in neointimal cells opposed to the internal elastic lamina. HDGF-positive medial and neointimal cells expressed

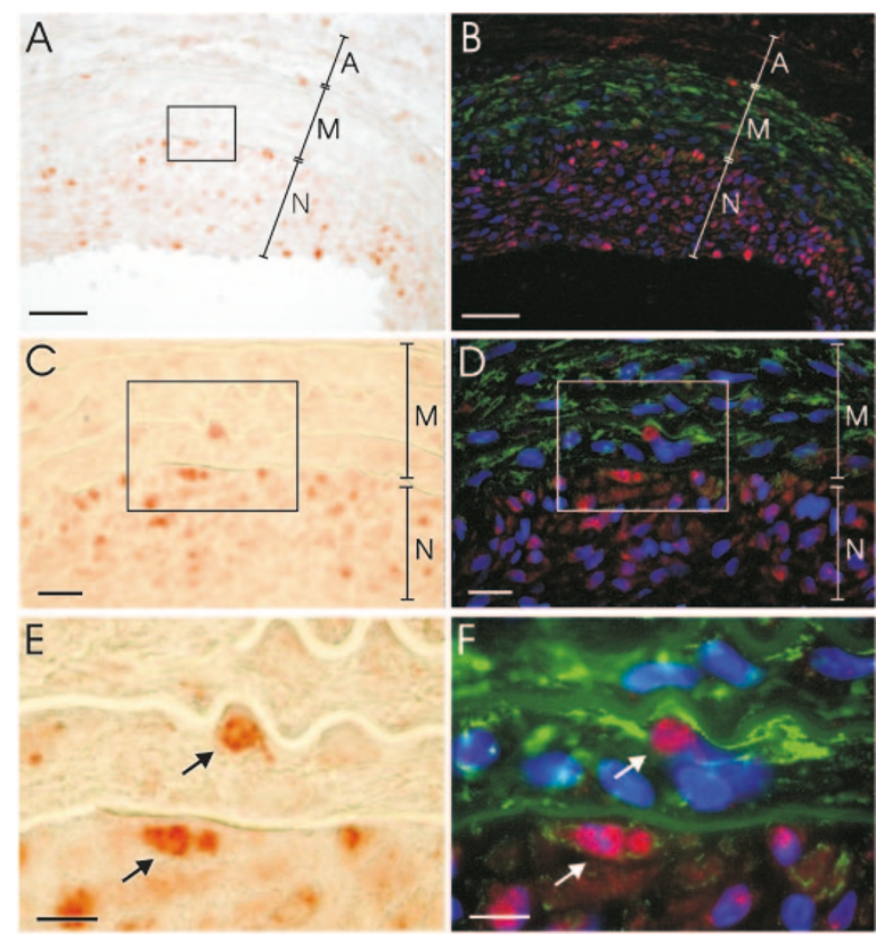

Figure 2. HDGF-positive medial and neointimal cells $7 \mathrm{~d}$ post injury lack $\alpha$ SMA immunostaining. $A, C$, and $E$ demonstrate HDGF at the light level (red). The companion fluorescent photomicrographs $B, D$, and $F$ show co-localization of HDGF (red), $\alpha$ SMA (green), and DNA (blue). The box in $C$ and $D$ is the area shown at higher magnification in $E$ and $F$. Arrows indicate HDGF-positive medial and neointimal cells that express little to no $\alpha \mathrm{SMA}$. $A$, adventitia; $M$, media; $N$, neointima. Magnification bar in $A$ and $B=100 \mu \mathrm{m}$, $C$ and $D=50 \mu \mathrm{m}, E$ and $F=20 \mu \mathrm{m}$. very little $\alpha$ SMA. HDGF-positive medial cells had a cytoplasm relatively devoid of $\alpha$ SMA, similar to HDGF positive neointimal cells (Fig. $2 F$ ) that have undergone phenotypic switching.

Viral overexpression of HDGF stimulates cell proliferation. Further assessment of a role for HDGF in SMC growth required the development of reagents to overexpress HDGF more efficiently. A replication-deficient type-5 adenovirus was prepared to express a GFP control or a GFP-HDGF fusion. Cells infected with the GFP control fluoresce green throughout the cytoplasm, whereas cells infected with a virus to express a GFP-HDGF fusion localize fluorescence to the nucleus (Fig. 3). This was consistent with our earlier work showing that HDGF is a nuclear-targeted protein in SMC. To determine the appropriate viral dose for mouse SMC, cells were infected with an increasing MOI, and cell lysates were immunoblotted with an anti-GFP antibody as a marker of viral expression (Fig. 3B). We found that our virus induces a robust response at a MOI as low as 10 . At a MOI of 100 or more, GFP expression was decreased due to a cytotoxic effect from the adenovirus. For our subsequent experiments, a MOI of 25 in mouse SMC was used with no cytotoxicity observed and as

A

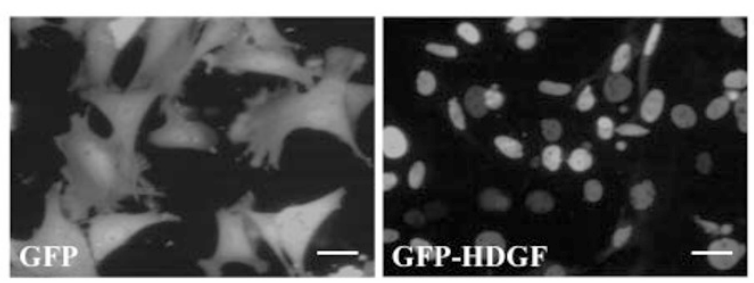

B
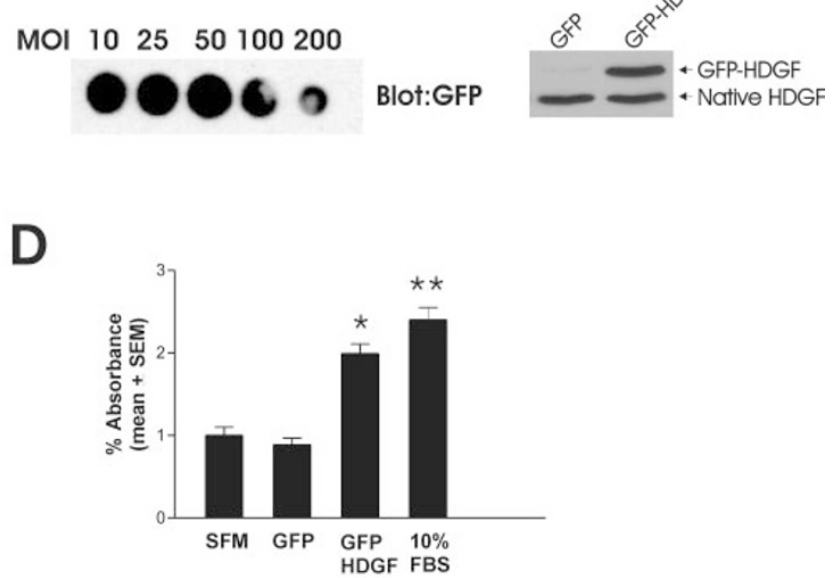

Figure 3. Adenoviral expression of a GFP-HDGF fusion in primary mouse SMC is nuclear targeted and mitogenic. (A) Global GFP expression in SMC infected with adenovirus (MOI 25) to express GFP (as a control) or nuclear expression with the GFP-HDGF fusions. $(B)$ Immunoblot for GFP to determine the MOI for experiments, a MOI of 25 was used for $A, C$, and $D$. (C) Western blot for HDGF of cell lysates from mouse SMC infected with the GFP and GFP-HDGF adenoviruses at an MOI of 25, demonstrated a doubling of the HDGF content. $(D)$ BrdU ELISA of SMC infected to express GFP or a GFP-HDGF fusion for $24 \mathrm{~h}$ then transferred to serum-free media (SFM) for 24 h. As controls, uninfected SMC were cultured in SFM or 10\% FBS before BrdU loading (12 h). $n=5$ for each group. * $p \leq 0.05$ GFP HDGF $v s$ GFP. $* * p \leq 0.0510 \%$ FBS vs SFM. Magnification bars $=20 \mu \mathrm{m}$. 
shown by Western blotting (Fig. $3 C$ ) results in doubling of the cellular HDGF content. To confirm that the adenovirus delivered HDGF was biologically active, we used BrdU incorporation as a marker of HDGF-stimulated cell proliferation. As shown in Figure $3 D$, adenoviral-delivered HDGF resulted in a significant 2-fold increase in BrdU incorporation compared with the GFP infected control.

Overexpression of HDGF increases SMC migration. Neointima formation occurs at least in part from activation and migration of SMC from the media to the intima (10-12). We performed modified Boyden chamber migration assays to evaluate whether HDGF stimulates in vitro SMC migration. Mouse SMC were infected with the adenovirus at an MOI of 25 (as in Fig. 3A) to express a GFP-HDGF fusion protein or GFP as a control and then plated for migration through a membrane after $12 \mathrm{~h}$ in SFM. At baseline in noninfected and GFP-expressing controls, there was very little migration of SMC to the serum containing media. Cells infected with GFP-HDGF adenovirus demonstrated marked migration through the membrane as evidenced by the many GFPpositive nuclei (Fig. 4) present on the underside of the membrane. When the number of migrating cells were counted, SMC expressing HDGF exhibited a 10 -fold increase in migration compared with control (20 versus $2, p<0.001)$ (Fig. 4).

HDGF is necessary for SMC proliferation and migration. To determine the significance of HDGF in SMC proliferation and migration in vitro, a shRNA delivered by a lentivirus specifically against mouse HDGF and human HDGF as a control was developed. As shown in Figure 5, lentiviral delivery of the HDGF shRNA in mouse SMC resulted in a $>75 \%$ decrease in HDGF protein levels by Western blotting $72 \mathrm{~h}$ after infection. Importantly, the human shRNA (hshRNA) had no effect on HDGF levels in mouse cells. With lentiviral shRNA expression we observed no effect on cell viability and all cells uniformly expressed GFP as a marker of infection. To determine whether HDGF gene silencing affects
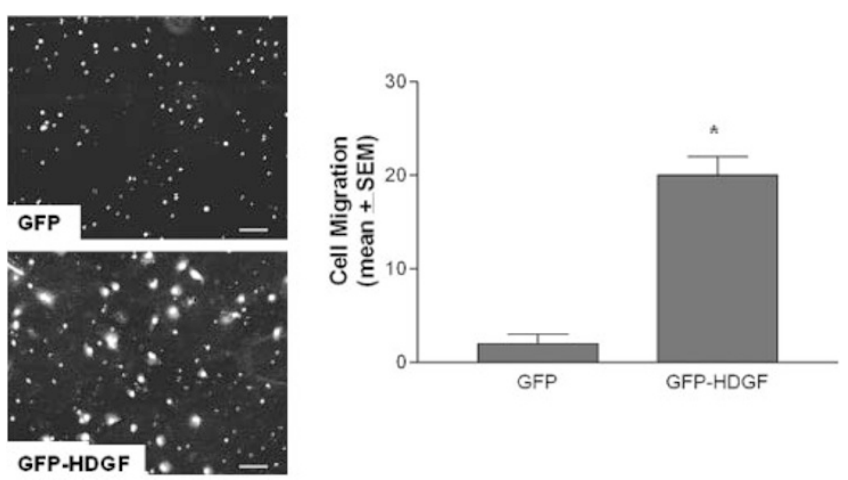

Figure 4. Adenoviral expression of HDGF in SMC increases migration. SMC infected to express GFP or a GFP-HDGF fusion (MOI 25) were allowed to migrate in a modified Boyden chamber for $12 \mathrm{~h}$ to SFM. Representative fluorescent photomicrographs on the left show the paucity of GFP control cells compared with GFP-HDGF-expressing cells that have migrated to the underside of the membrane. On the right is a graphic representation comparing the number of migrating GFP- or GFP-HDGF-expressing cells $(n=5$ membranes in two separate experiments). $* p<0.001$. Magnification bars $=$ $50 \mu \mathrm{m}$.
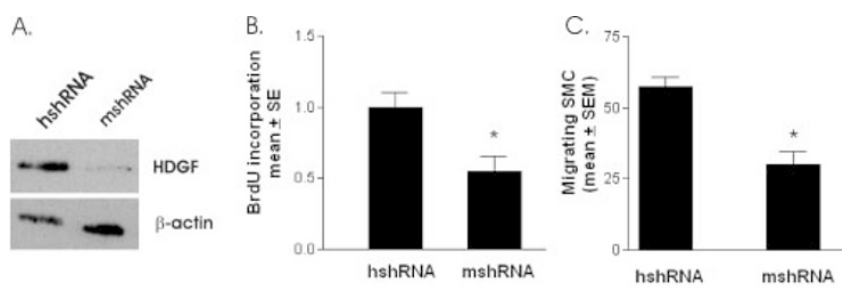

Figure 5. HDGF gene silencing in SMC blunts proliferation and migration. Primary mouse aortic SMC were infected with a lentivirus to express a human- (hshRNA) or mouse- (mshRNA) specific HDGF short hairpin interfering RNA. (A) Western blotting for HDGF $48 \mathrm{~h}$ after infection demonstrated a specific decrease in HDGF protein with the mshRNA and no effect with the hshRNA. ( $B)$ BrdU incorporation $(12 \mathrm{~h})$ as measured by ELISA $(n=5)$ to measure the effect of HDGF gene silencing on replication. (C) The number of SMC migrating cells ( $12 \mathrm{~h}$ ) after $48 \mathrm{~h}$ of infection to express HDGF shRNAs ( $n=5$ membranes) in media with $10 \%$ FBS. ${ }^{*} p<0.05$.

SMC proliferation in media with $10 \%$ FBS, SMC infected to express the mouse or human HDGF shRNA lentivirus as a control were plated after $4 \mathrm{~d}$ into a 96-well plate with BrdU incorporation for $12 \mathrm{~h}$ determined by ELISA. As shown in Figure 5, HDGF gene silencing resulted in a significant decrease in BrdU uptake in response to growth in 10\% FBS compared with controls.

To examine the role of HDGF in mediating SMC migration, mouse SMC infected to express mouse or human HDGF shRNA or empty virus for $4 \mathrm{~d}$ were also plated to migration membranes to measure migration rates over $12 \mathrm{~h}$. As shown in Figure 5 the migration of SMC in $10 \%$ FBS is significantly decreased in cells with HDGF gene silencing.

\section{DISCUSSION}

The response to vascular injury that occurs with angioplasty and stenting of arteries in children and adults is a complex cascade of events ultimately leading to SMC proliferation and migration with resultant neointimal hyperplasia. Peptide growth factors (e.g., PDGF, b-FGF) have previously been linked to neointimal formation, and this study represents the first evidence that HDGF, a novel nuclear-targeted protein, stimulates SMC migration. Previous work has demonstrated that HDGF is a potent SMC mitogen, and it is now apparent that HDGF plays an additional role in the SMC response to injury.

Immunohistochemical staining of carotid artery sections after injury confirmed that HDGF is expressed in the media at $3 \mathrm{~d}$ and represents an early and sensitive marker of injury as baseline expression of HDGF in the arterial media is very low (6). HDGF expression is even greater in the forming neointima at $7 \mathrm{~d}$ after injury with $97 \%$ of neointimal nuclei HDGF positive. Double immunostaining for HDGF and PCNA in our study at $3 \mathrm{~d}$ post injury revealed very little to no expression of PCNA in the carotid media, whereas at $7 \mathrm{~d}$ there was significant co-expression of HDGF in the highly proliferative neointima. These PCNA immunostaining results are consistent with Mills et al. (13), which have shown low levels of PCNA, only in adventitial cells, at $1-3 \mathrm{~d}$ post injury with most of the cells in the neointima PCNA positive by $7 \mathrm{~d}$. However, it was not possible in this study to determine whether HDGF was 
expressed before PCNA and thus a trigger for PCNA expression. It is likely that the mechanism of increased HDGF expression in the arterial well and its downstream effects are complex. Previously, we have shown that unlike serum, PDGF and thrombin do not regulate HDGF expression in SMC (6). Yet, increased HDGF expression leads to increased cell proliferation and a loss of HDGF expression (gene silencing) results in SMC with a decreased proliferative capacity. Taken together, this data places HDGF as part of a pathway activated after arterial injury to work in consort with other factors to stimulate SMC proliferation after injury.

Following SMC proliferation in the media, chemotactic migration of SMC from the media to the intima of the vessel wall is a critical step in the neointimal lesion that results after vascular injury $(14,15)$. In vitro studies of cell migration using modified Boyden chamber migration assays established that cells overexpressing HDGF exhibited a 10-fold increase in cell migration when compared with control. This increase in migration over $12 \mathrm{~h}$ is unlikely to result from increased proliferation because, under optimal conditions with serumcontaining media, SMC expressing HDGF have a cell doubling time of $48 \mathrm{~h}$ (6). This increase in SMC migration was complemented by gene silencing experiments in which decreased expression of endogenous HDGF blunted the normal migratory response of SMC in culture. In support of HDGF playing a broad role in cell migration, HDGF was identified as one of several genes up-regulated in retinal pigment epithelial cells stimulated to migrate in a wound assay (16). Our observation of HDGF expression in medial SMC after injury that were not proliferating suggests that HDGF could at least be a marker of SMC that have undergone phenotypic switching and are activated to migrate. Taken together, these studies demonstrate a role for HDGF in regulating SMC migration.

The mechanism of HDGF-stimulated cell migration is unclear. Increased expression of HDGF in vitro did not alter MMP activity by zymography or MMP-9 levels by Western blot (data not shown) as a mechanism. Whether HDGF regulates MMP expression and activity within the arterial wall is yet to be determined.

The SMC of the arterial medial layer have been shown to contain subpopulations of cells that, when stimulated by vascular injury, are induced to proliferate (17-20). This change in phenotype to become a proliferative cell is associated with a decrease in contractile phenotype as described by decreased, $\alpha$ SMA, smooth muscle myosin heavy chain and SM22 $\alpha$ $(10,11)$, is termed phenotypic switching. We observed that HDGF expression in specific medial cells is seen as early as $3 \mathrm{~d}$ after injury with expression in both medial and neointimal cells at $7 \mathrm{~d}$. We observed that HDGF-positive medial and neointimal cells $7 \mathrm{~d}$ after injury were associated with a decline in $\alpha$ SMA expression. It has been shown by Thyberg et al. (21) using immunohistochemistry coupled with electron microscopy that SMC near the neointima express little $\alpha$ SMA (as shown by the HDGF-positive neointimal cells in Fig. 2), have cytoplasmic features of increased synthetic ability, and were observed to migrate through the internal elastic lamina to the neointima. Therefore, supporting the idea that linking HDGF expression to a decrease in $\alpha \mathrm{SMA}$ identifies a subpopulation of arterial medial cells that can migrate and proliferate in response to injury. It is possible that at least a portion of the HDGF-positive neointimal and medial cells may not be SMC but represent extravascular leukocytes that are known to populate the arterial wall after injury. However, as shown by Gotoh et al. (22) these cells make up less than $0.2 \%$ of medial or neointimal cells at 3 and $7 \mathrm{~d}$ post injury. Therefore, the high cellular expression of HDGF by medial and neointimal cells is unlikely to be due predominately to leukocyte infiltration. This suggests that HDGF expression is a marker for a subpopulation of SMC that have undergone phenotypic switching.

It is estimated that $20-30 \%$ of treated atherosclerotic lesions occlude as a result of restenosis, even with drug-eluting stents $(1,2)$. Therefore, approaches to inhibit restenosis due to neointimal formation will prove crucial in improving the long-term efficacy of vascular interventions such as balloon angioplasty. Although further studies are necessary to elucidate the precise signaling mechanisms that promote SMC proliferation and migration, our results indicate that HDGF is expressed early in the vascular wall after injury and is a SMC mitogen that also stimulates SMC migration. As such, HDGF represents a potential therapeutic target for neointimal hyperplasia after balloon angioplasty.

\section{REFERENCES}

1. Cutlip DE, Chauhan MS, Baim DS, Ho KK, Popma JJ, Carrozza JP, Cohen DJ, Kuntz RE 2002 Clinical restenosis after coronary stenting: perspectives from multicenter clinical trials. J Am Coll Cardiol 40:2082-2089

2. Indolfi C, Mongiardo A, Curcio A, Torella D 2003 Molecular mechanisms of in-stent restenosis and approach to therapy with eluting stents. Trends Cardiovasc Med 13:142-148

3. Post M, Waltenberger J 2005 Modulation of growth factor action in the cardiovascular system. Cardiovasc Res 65:547-549

4. Levitzki A 2005 PDGF receptor kinase inhibitors for the treatment of restenosis. Cardiovasc Res 65:581-586

5. Nakamura H, Izumoto Y, Kambe H, Kuroda T, Mori T, Kawamura K, Yamamoto H, Kishimoto T 1994 Molecular cloning of complementary DNA for a novel human hepatoma-derived growth factor. Its homology with high mobility group-1 protein. J Biol Chem 269:25143-25149

6. Everett AD, Lobe DR, Matsumura ME, Nakamura H, McNamara CA 2000 Hepatoma-derived growth factor stimulates smooth muscle cell growth and is expressed in vascular development. J Clin Invest 105:567-575

7. Everett AD, Narron JV, Stoops T, Nakamura H, Tucker A 2004 Hepatoma-derived growth factor is a pulmonary endothelial cell-expressed angiogenic factor. Am J Physiol Lung Cell Mol Physiol 286:L1194-L1201

8. Hardy S, Kitamura M, Harris-Stansil T, Dai Y, Phipps ML 1997 Construction of adenovirus vectors through Cre-lox recombination. J Virol 71:1842-1849

9. Rubinson DA, Dillon CP, Kwiatkowski AV, Sievers C, Yang L, Kopinja J, Zhang M, McManus MT, Gertler FB, Scott ML, Van Parijs L 2003 A lentivirus-based system to functionally silence genes in primary mammalian cells, stem cells and transgenic mice by RNA interference. Nat Genet 33:401-406

10. Owens GK, Kumar MS, Wamhoff BR 2004 Molecular regulation of vascular smooth muscle cell differentiation in development and disease. Physiol Rev 84:767-801

11. Owens GK 1995 Regulation of differentiation of vascular smooth muscle cells. Physiol Rev 75:487-517

12. Taylor JM, Mack CP, Nolan K, Regan CP, Owens GK, Parsons JT 2001 Selective expression of an endogenous inhibitor of FAK regulates proliferation and migration of vascular smooth muscle cells. Mol Cell Biol 21:1565-1572

13. Mills CJ, Northrup JL, Hullinger TG, Simmons CA, Shebuski RJ, Jones DA 1996 Temporal expression of c-fos mRNA following balloon injury in the rat common carotid artery. Cardiovasc Res 32:954-961

14. Bilato C, Pauly RR, Melillo G, Monticone R, Gorelick-Feldman D, Gluzband YA, Sollott SJ, Ziman B, Lakatta EG, Crow MT 1995 Intracellular signaling pathways required for rat vascular smooth muscle cell migration. Interactions between basic fibroblast growth factor and platelet-derived growth factor. J Clin Invest 96:19051915

15. Boehm M, Nabel E 2001 Cell cycle and cell migration: new pieces to the puzzle. Circulation 103:2879-2881

16. Singh S, Zheng JJ, Peiper SC, Mclaughlin BJ 2001 Gene expression profile of ARPE-19 during repair of the monolayer. Graefes Arch Clin Exp Ophthalmol 239:946-951 
17. Clowes AW, Reidy MA, Clowes MM 1983 Mechanisms of stenosis after arterial injury. Lab Invest 49:208-215

18. Clowes AW, Reidy MA, Clowes MM 1983 Kinetics of cellular proliferation after arterial injury. I. Smooth muscle growth in the absence of endothelium. Lab Invest 49:327-333

19. Clowes AW, Clowes MM, Reidy MA 1986 Kinetics of cellular proliferation after arterial injury. III. Endothelial and smooth muscle growth in chronically denuded vessels. Lab Invest 54:295-303

20. Clowes AW, Clowes MM, Fingerle J, Reidy MA 1989 Kinetics of cellular prolif- eration after arterial injury. V. Role of acute distension in the induction of smooth muscle proliferation. Lab Invest 60:360-364

21. Thyberg J, Blomgren K, Roy J, Tran PK, Hedin U 1997 Phenotypic modulation of smooth muscle cells after arterial injury is associated with changes in the distribution of laminin and fibronectin. J Histochem Cytochem 45:837-846

22. Gotoh R, Suzuki J, Kosuge H, Kakuta T, Sakamoto S, Yoshida M, Isobe M 2004 E-selectin blockade decreases adventitial inflammation and attenuates intimal hyperplasia in rat carotid arteries after balloon injury. Arterioscler Thromb Vasc Biol 24:2063-2068 